\title{
Tail homogeneity of invariant measures of multidimensional stochastic recursions in a critical case
}

\author{
Konrad Kolesko
}

Received: 19 May 2011 / Revised: 14 March 2012 / Published online: 12 June 2012

(C) The Author(s) 2012. This article is published with open access at Springerlink.com

\begin{abstract}
We consider the stochastic recursion $X_{n+1}=M_{n+1} X_{n}+Q_{n+1},(n \in \mathbb{N})$, where $Q_{n}, X_{n} \in \mathbb{R}^{d}, M_{n}$ are similarities of the Euclidean space $\mathbb{R}^{d}$ and $\left(Q_{n}, M_{n}\right)$ are i.i.d. We study asymptotic properties at infinity of the invariant measure for the Markov chain $X_{n}$ under assumption $\mathbb{E}[\log |M|]=0$ i.e. in the so called critical case.
\end{abstract}

Keywords Random walk - Affine group - Tail homogeneity · Invariant measure

Mathematics Subject Classification (2000) 60B15

\section{Introduction}

We consider the vector space $V=\mathbb{R}^{d}$ endowed with the scalar product $\langle u, v\rangle=$ $\sum_{i=1}^{d} u_{i} v_{i}$ and the norm $|u|=\left(\sum_{i=1}^{d}\left|u_{i}\right|^{2}\right)^{\frac{1}{2}}$. Let $\operatorname{Aff}(V)=V \rtimes \operatorname{GL}(V)$ be the group of affine mappings of $V$, i.e. Aff $(V)$ is the semi-direct product of the linear group $\operatorname{GL}(V)$ and the group of translations of $V$. The action of the group $\operatorname{Aff}(V)$ on $V$ is given by the following formula

$$
h u=g u+b, \quad h=(b, g) \in \operatorname{Aff}(\mathrm{V}), u \in V .
$$

Given a probability measure $\mu$ on $\operatorname{Aff}(V)$ and $u \in V$, we consider the recurrence relation with random coefficients

This research project has been partially supported by MNiSW grant N N201 610740 and also by FWF grant FWF-P19115-N18.

K. Kolesko $(\bowtie)$

Instytut Matematyczny, Uniwersytet Wrocławski,

pl. Grunwaldzki 2/4, 50-384 Wrocław, Poland

e-mail: kolesko@math.uni.wroc.pl 


$$
\begin{aligned}
& X_{0}^{u}=u, \\
& X_{n}^{u}=M_{n} X_{n-1}^{u}+Q_{n},
\end{aligned}
$$

where the pairs $\left(Q_{n}, M_{n}\right) \in \operatorname{Aff}(V)$ are independent with law $\mu$.

The random process $X_{n}^{u}$ has been studied mostly in the one dimensional settings i.e. when $V=\mathbb{R}$ and $(Q, M) \in \mathbb{R} \rtimes \mathbb{R}^{+}$. It is well known that if $\mathbb{E} \log M<0$ and $\mathbb{E} \log ^{+}|Q|<\infty$, then there exists a unique stationary measure $v$ of the Markov chain $\left\{X_{n}^{u}\right\}$, i.e. a probability measure $v$ on $\mathbb{R}$ such that

$$
v(f)=\mu * v(f)=\int_{\operatorname{Aff}(V)} \int_{V} f(a u+b) v(\mathrm{~d} u) \mu(\mathrm{d} a \mathrm{~d} b)
$$

for any bounded function $f$ on $\mathbb{R}$. One of the most significant result is due to Kesten [14] (see also Goldie [11]), who under a number of further assumptions, the main being the existence of $\alpha>0$ such that $\mathbb{E} M^{\alpha}=1$, proved that the measure $v$ is $\alpha$-regularly varying, i.e.

$$
\lim _{t \rightarrow \infty} t^{\alpha} \nu\{|u|>t\}=C_{+}
$$

Recently in a number of papers also the critical case, $\mathbb{E} \log M=0$, has been investigated. The main result is due to Babillot et al. [1], who proved that there exists a unique invariant Radon measure $v$ of the process $\left\{X_{n}^{u}\right\}$, i.e. the measure satisfying (2), but in this case it is infinite on $\mathbb{R}$. Its behaviour was described by Buraczewski [6] and Brofferio et al. [5]. They proved the Kesten theorem in the critical case:

$$
\lim _{t \rightarrow \infty} v\left\{r_{1} t<|u| \leq r_{2} t\right\}=C_{+} \log \left(r_{2} / r_{1}\right)
$$

The multidimensional situation, $d>1$, is much harder. In the contracting case it was studied by Kesten [14] and later on by Le Page [15] and Guivarc'h [13], who applying quite involved techniques obtained results similar to (3). However the critical case, when the top Lapunov exponent is zero, seems to be still not well understood and up to now has been studied only in very restrictive settings. Existence and uniqueness of the invariant measure was proved by Brofferio [3,4] in a particular case, when $M$ are either upper triangular matrices or they are of the form $D K, D$ being a dilation and $K$ an element of $\mathrm{O}(d)$ (in fact this was proved only for $K=I d$, but exactly the same proof is valid in the general case). Behaviour of $v$ at infinity was studied in [5] only if $M$ is a dilation matrix and then the result is reduced indeed to a one dimensional case.

In this paper we assume that $M_{n}$ belongs to the similarity group $G$ of $V$, i.e. the group of elements $g$ of GL( $V)$ satisfying

$$
|g u|=|g||u|,
$$

for every $u \in V$. The group of similarities $G$ is the direct product of $\mathbb{R}_{+}^{*}$ and the orthogonal group $\mathrm{O}(V)$. Consequently any element $g$ of $G$ can be uniquely written in the form $g=t k$ for $t \in \mathbb{R}_{+}^{*}$ and $k \in \mathrm{O}(V)$. By $\bar{\mu}$ we denote the projection of 
the measure $\mu$ onto $G$ i.e. the law of the random variable $M_{n}$. Let $G_{\mu}$ be the closed subgroup of $G$ generated by the support of $\bar{\mu}$.

The structure of $G_{\mu}$ is well known (see e.g. Introduction in [7]). If we assume that $G_{\mu}$ is not contained in the orthogonal group $\mathrm{O}(V)$, then there are two cases: either the projection of $G_{\mu}$ into $\mathbb{R}_{+}^{*}$ is $\mathbb{R}_{+}^{*}$ (non-lattice case) or its subgroup isomorphic to $\mathbb{Z}$ (lattice case).

It turns out that the center $Z_{\mu}$ of the group $G_{\mu}$ is relatively large i.e. $G_{\mu} / Z_{\mu}$ is compact. In the first case one can find a subgroup $A_{\mu}$ of $G_{\mu}$ that is isomorphic to $\mathbb{R}_{+}^{*}$ and is contained in the center $Z_{\mu}$. It yields that $G_{\mu}$ is isomorphic to $O_{\mu} \times A_{\mu}$, for $O_{\mu}=G_{\mu} \cap \mathrm{O}(V)$. In the second case, in generally, it is not true that $G_{\mu}$ is isomorphic to the direct product of $O_{\mu}$ and a projection of $G_{\mu}$ of $\mathbb{R}_{+}^{*}$. However, one can find $c \in G_{\mu}$ and $A_{\mu}=\langle c\rangle$ such that $G_{\mu}=O_{\mu} \rtimes A_{\mu}$ and $A_{\mu}$ contains a central subgroup of $G_{\mu}$ as a finite index subgroup (see Proposition A.1 in Appendix in [7] for more details). In both cases there exists a compact subset $K_{\mu} \subseteq G_{\mu}$ such that $G_{\mu}=Z_{\mu} K_{\mu}$.

Now, similarly to [8], we introduce the generalized polar coordinates. First set $\Sigma_{1}=S_{1}=\{v \in V:|v|=1\}$ in the lattice case or $\Sigma_{1}=\{v \in V: 1 \leq|v|<|c|\}$. The set $\Sigma_{1}$ is the fundamental domain for the action $A_{\mu}$ on $V \backslash\{0\}$. Any element $v \in V$ can be written in a unique way as $v=a(v) \bar{v}$ for $a(v) \in A_{\mu}$ and $\bar{v} \in \Sigma_{1}$.

Since $G$ is unimodular, as well as any its closed subgroup, the left and the right Haar measure coincide. By $m_{G}$ (resp. $m_{G_{\mu}}, m_{A_{\mu}}$ ) we denote the Haar measure on $G$ (resp. on $\left.G_{\mu}, A_{\mu}\right)$ normalized in such a way that $m_{G}(\{g: 1 \leq|g|<t\})=\log t$, where $t$ is an arbitrary positive number in the non-lattice case or $|c|$ in the lattice. For simplicity we shall write just d $g$ instead of $m_{G_{\mu}}$. By $H=V \rtimes G$ will denote the affine group with similarity matrix.

Under this settings i.e. when $M$ is a similarity matrix, Eq. (1) has been recently studied in the contracting case $\mathbb{E} \log |M|<0$ by Buraczewski et al. [7,8]. They described the tail of the stationary measure [8] and then proved limit theorems related to partial sums $X_{1}^{u}+\cdots+X_{n}^{u}$, [7].

Here we study the critical case, i.e. from now we assume that $\mathbb{E}[\log |M|]=0$. Existence and uniqueness of the invariant measure $v$ in the critical case was proved by Brofferio [3] under the assumptions

$$
\begin{aligned}
& \forall u \mathbb{P}[M u+Q=u]<1, \quad \mathbb{P}[|M|=1]<1, \\
& \mathbb{E}\left[(\log |M|)^{2}+\left(\log ^{+}|Q|\right)^{2+\varepsilon}\right]<\infty \quad \text { for some } \varepsilon>0, \\
& \mathbb{E}[\log |M|]=0 .
\end{aligned}
$$

The main purpose of the present paper is to describe the behaviour of $v$ at infinity. Our main result is

Theorem 1 Assume that hypotheses (A1)-(A3) are satisfied and moreover

$$
\mathbb{E}\left[|M|^{\delta}+|M|^{-\delta}+|Q|^{\delta}\right]<\infty,
$$

then there exists a positive Radon measure $\Lambda$ on $V \backslash\{0\}$ such that the family of measure $\left\{\delta_{(0, g)} * v\right\}_{g \in G_{\mu}}$ restricted to $V \backslash\{0\}$ satisfies 


$$
\lim _{|g| \rightarrow 0, g \in G_{\mu}} \delta_{(0, g)} * v=\Lambda
$$

where the convergence is in the vague topology i.e. for any $\phi \in C_{c}(V \backslash\{0\})$.

$$
\lim _{|g| \rightarrow 0, g \in G_{\mu}} \int_{V \backslash\{0\}} \phi(g u) v(\mathrm{~d} u)=\int_{V \backslash\{0\}} \phi(u) \Lambda(\mathrm{d} u) .
$$

Moreover $\Lambda$ is $G_{\mu}$-invariant. That is there exists a finite positive $O_{\mu}$-invariant measure $\sigma_{\mu}$ on $\Sigma_{1}$ such that

$$
\Lambda=m_{A_{\mu}} \otimes \sigma_{\mu}
$$

In particular we obtain new results in the one dimension, when $M_{n}$ and $Q_{n}$ are just scalars and admit both positive and negative values. In this case we have

Corollary 1 Under the assumptions of Theorem 1 the invariant measure $v$ in one dimensional non lattice case satisfies $v\left( \pm t r_{1}, \pm t r_{2}\right) \sim C_{ \pm} \log \frac{r_{2}}{r_{1}}$ as $t$ goes to infinity. Moreover, if $\mathbb{P}[M<0]>0$, then $C_{-}=C_{+}$.

We obtain also new results concerning the lattice case (compare [5]):

Corollary 2 Assume that the hypotheses of Theorem 1 are satisfied and moreover $M \in\left\{c^{n}: n \in \mathbb{Z}\right\}$ almost surely for some $c>1$, then

$$
\lim _{n \rightarrow \infty} \int_{V} \phi\left(u / c^{n}\right) v(\mathrm{~d} u)=\sum_{k \in \mathbb{Z}_{1 \leq|u|<c}} \int_{1} \phi\left(c^{k} u\right) \sigma_{\mu}(\mathrm{d} u),
$$

for any $\phi \in C_{c}(V \backslash\{0\})$.

In order to prove the theorem first we proceed as in [5]. We prove that there exists compactly supported function $\Phi$ such that $\delta_{(0, g)} * v(\Phi)>0$ for $|g|<1$ and $\frac{\delta_{(0, g h)} * \nu(\Phi)}{\delta_{(0, g)} * \nu(\Phi)} \rightarrow 1$ as $|g| \rightarrow 0$. Moreover, the family of measures $\frac{\delta_{(0, g)} * \nu}{\delta_{(0, g)} * \nu(\Phi)}$ is vaguely relatively compact when $|g| \leq 1$. Next given $\phi \in C_{c}(V \backslash\{0\})$ we define the function $f_{\phi}$ on $G$ :

$$
f_{\phi}(g)=\delta_{(0, g)} * v(\phi)=\int_{V} \phi(g u) v(\mathrm{~d} u)
$$

and we consider the Poisson equation on $G$

$$
\mathbb{E}\left[f_{\phi}(g M)\right]=f_{\phi}(g)+\psi_{\phi}(g) \text {. }
$$

In the one dimensional setting when $G=\mathbb{R}^{+}$, in $[5,6]$, the Poisson equation was solved and the main result was deduced from the explicit form of positive solutions, which is known if $G$ is abelian [16]. However if $G \neq \mathbb{R}^{+}$and it is not abelian we 
are still able to describe behaviour at infinity of solutions of the Poisson equation. For this purpose we apply a technique due to Durrett and Liggett [9] based on the duality lemma, which allows to modify the Poisson equation and to consider $f_{\phi}$ as a solution of the classical renewal equation (for a random walk with a drift). All the details will be figure out in the next section.

In this paper we will slightly abuse the notation considering $\phi \in C_{c}(V \backslash\{0\})$ (resp. $\left.C_{c}^{1}(V \backslash\{0\})\right)$ also as a function $C_{c}(V)\left(\operatorname{resp} . C_{c}^{1}(V)\right)$ with $0 \notin \operatorname{supp} \phi$.

\section{Proof of Theorem 1}

\subsection{Some auxiliary results}

First we prove here some estimates of the measure $v$. We follow the proof of Theorem 2.1 in [5]. However, since now we deal with nonabelian group $G_{\mu}$ acting on a multidimensional vector space $V$ and some of the estimates are very delicate, for the reader convenience we present all the details, emphasizing the steps where nonabelianity plays a role.

Lemma 1 There exists a radial function $\Phi \in C_{c}^{1}(V \backslash\{0\})$ such that the function $f_{\Phi}(g)=\delta_{(0, g)} * v(\Phi)$ is positive whenever $|g|<1$ and the family of measures

$$
\frac{\delta_{(0, g)} * v}{f_{\Phi}(g)}
$$

restricted to $V \backslash\{0\}$ is vaguely relatively compact as $|g|<1$.

The function $\Phi$ can be chosen in such a way that the integral $\int_{G_{\mu}} \Phi(g u) \mathrm{d} g$ is independent of $u \in V \backslash\{0\}$.

Proof First we prove that there exists $R$ large enough such that $\delta_{(0, g)} * v(\{1 / R \leq u \leq$ $R\})>0$ for $|g|<1$. For any $\alpha$ and $\beta$, consider the annulus $C(\alpha, \beta)=\{u \in V: \alpha \leq$ $|u| \leq \beta\}$. It is easy to check that for all $(b, a) \in H$ the following implication holds

$$
u \in C\left(\frac{\alpha+|b|}{|a|}, \frac{\beta-|b|}{|a|}\right) \Rightarrow a u+b \in C(\alpha, \beta) .
$$

Since $v$ in invariant with respect to $\mu^{n}$, the $n$-th convolution power of the measure $\mu$, we have

$$
\begin{aligned}
\delta_{(0, g)} * v(C(\alpha, \beta)) & =\int_{V} \int_{H} \mathbf{1}_{C(\alpha, \beta)}(g(a x+b)) \mu^{n}(\mathrm{~d} b, \mathrm{~d} a) v(d x) \\
& \geq \int_{V} \int_{H} \mathbf{1}_{C\left(|g|^{-1} \alpha,|g|^{-1} \beta\right)}(a x+b) \mathbf{1}_{U}(b, a) \mu^{n}(\mathrm{~d} b, \mathrm{~d} a) v(d x) \\
& \geq \mu^{* n}(U) v\left(C\left(\max _{(b, a) \in U} \frac{|g|^{-1} \alpha+|b|}{|a|}, \min _{(b, a) \in U} \frac{|g|^{-1} \beta-|b|}{|a|}\right)\right) .
\end{aligned}
$$


Since $\mathbb{P}[|M|=1]<1$, there are $h_{-}=\left(b_{-}, a_{-}\right)$and $h_{+}=\left(b_{+}, a_{+}\right)$belonging to the support of $\mu$ such that $\left|a_{-}\right|<1<\left|a_{+}\right|$. For fixed $g \in G_{\mu}$ with $|g|<1$, there exists a natural number $n$ such that $\left|a_{+}\right|^{n-1} \leq|g|^{-1} \leq\left|a_{+}\right|^{n}$. Clearly, if $h^{n}=\left(b\left(h^{n}\right), a\left(h^{n}\right)\right)$ is the $n$-th power of an element $h=(b, a) \in H$ then $a\left(h^{n}\right)=a^{n}$ and $b\left(h^{n}\right)=$ $\sum_{i=0}^{n-1} a^{i} b$, hence

$$
\left|b\left(h^{n}\right)\right| \leq \sum_{i=0}^{n-1}|a|^{i}|b|=\frac{|a|^{n}-1}{|a|-1}|b| .
$$

The $\gamma$-neighbourhood of an element $h \in H$ is the open set $U_{\gamma}$ defined by

$$
U_{\gamma}(h)=\left\{(b, a) \in H: e^{-\gamma}<|a \cdot a(h)|<e^{\gamma},|b-b(h)|<\gamma\right\} .
$$

Since $h_{+}$is in the support of $\mu$ it follows that for any $\gamma>0, \mu^{n}\left(U_{\gamma}\left(h_{+}^{n}\right)\right)>0$ and moreover for $(b, a) \in U_{\gamma}\left(h_{+}^{n}\right)$, by (8) we have

$$
\begin{aligned}
\frac{|g|^{-1} \alpha+|b|}{|a|} & \leq \frac{\left|a\left(h_{+}\right)\right|^{n} \alpha+\left|b\left(h_{+}^{n}\right)\right|+\gamma}{\left|a\left(h_{+}\right)\right|^{n} e^{-\gamma}} \\
& \leq e^{\gamma}\left(\alpha+\frac{\left(\left|a\left(h_{+}\right)\right|^{n}-1\right)\left|b\left(h_{+}\right)\right|}{\left.\left|\left(a\left(h_{+}\right) \mid-1\right)\right| a\left(h_{+}\right)\right|^{n}}+\frac{\gamma}{\left|a\left(h_{+}\right)\right|^{n}}\right) \\
& =e^{\gamma}\left(\alpha+\frac{\left|b\left(h_{+}\right)\right|}{\left|a\left(h_{+}\right)\right|-1}+\gamma\right)=: \alpha^{\prime}
\end{aligned}
$$

and on the other side

$$
\begin{aligned}
\frac{|g|^{-1} \beta-|b|}{|a|} & \geq \frac{\left|a\left(h_{+}\right)\right|^{n-1} \beta-\left|b\left(h_{+}^{n}\right)\right|-\gamma}{\left|a\left(h_{+}\right)\right|^{n} e^{\gamma}} \\
& \geq e^{-\gamma}\left(\frac{\beta}{\left|a\left(h_{+}\right)\right|}-\frac{\left|b\left(h_{+}\right)\right|}{\left|a\left(h_{+}\right)\right|-1}-\gamma\right)=: \beta^{\prime} .
\end{aligned}
$$

Thus is $u \in C\left(\alpha^{\prime}, \beta^{\prime}\right)$, then $a u+b \in C(\alpha, \beta)$ for any $(b, a) \in U_{\gamma}\left(h_{+}^{n}\right)$. One can see that $\alpha^{\prime}$ is decreasing when $\alpha$ does and $\beta^{\prime}$ goes to infinity when $\beta$ does. Therefore, since $v$ is a Radon measure with infinite mass, there exists sufficiently large $R$ such that taking $\frac{1}{\alpha}=\beta=R$ and appropriate $\alpha^{\prime}$ and $\beta^{\prime}$, by (7) we get

$$
\delta_{(0, g)} * v(C(1 / R, R)) \geq \mu^{n}\left(U_{\gamma}\left(h_{+}^{n}\right)\right) v\left(C\left(\alpha^{\prime}, \beta^{\prime}\right)\right)>0
$$

for any $g$ with $|g| \leq 1$ and $n=\left\lfloor(|g| \log |u|)^{-1}\right\rfloor-1$.

Without any loss of generality we may assume $R>2 \max \left\{1 /\left|a_{-}\right|,\left|a_{+}\right|\right\}$. We consider a family of compact sets $K_{ \pm}^{n}=C\left(\frac{2}{R}\left|a_{ \pm}\right|^{-n}, \frac{R}{2}\left|a_{ \pm}\right|^{-n}\right)$ covering $V \backslash\{0\}$. 
Observe that for $\gamma<\log (4 / 3),(b, a) \in U_{\gamma}\left(h_{ \pm}^{n}\right)$ and $|g|<\left[2 R\left(\left|b\left(h_{ \pm}^{n}\right)\right|+\gamma\right)\right]^{-1}$ we have:

$$
\begin{aligned}
\frac{|g|^{-1} / R+|b|}{|a|} & \leq|g|^{-1} e^{\gamma} \frac{1 / R+|g|\left(\left|b\left(h_{ \pm}^{n}\right)\right|+\gamma\right)}{\left|a_{ \pm}\right|^{n}} \\
& \leq|g|^{-1} \frac{2\left|a_{ \pm}\right|^{-n}}{R}\left(e^{\gamma} \frac{1+|g| R\left(\left|b\left(h_{ \pm}^{n}\right)\right|+\gamma\right)}{2}\right) \leq|g|^{-1} \frac{2\left|a_{ \pm}\right|^{-n}}{R} .
\end{aligned}
$$

On the other hand

$$
\begin{aligned}
\frac{|g|^{-1} R-|b|}{|a|} & \geq|g|^{-1} e^{-\gamma} \frac{R-|g|\left(\left|b\left(h_{ \pm}^{n}\right)\right|+\gamma\right)}{\left|a_{ \pm}\right|^{n}} \\
& \geq|g|^{-1} \frac{R\left|a_{ \pm}\right|^{-n}}{2} \cdot 2 e^{-\gamma}\left(1-\frac{|g|\left(\left|b\left(h_{ \pm}^{n}\right)\right|+\gamma\right)}{R}\right) \geq|g|^{-1} \frac{R\left|a_{ \pm}\right|^{-n}}{2} .
\end{aligned}
$$

Therefore

$$
C\left(\frac{|g|^{-1} / R+|b|}{|a|} ; \frac{|g|^{-1} R-|b|}{|a|}\right) \supseteq C\left(|g|^{-1} \frac{2}{\left|a_{ \pm}\right|^{n} R} ;|g|^{-1} \frac{R}{2\left|a_{ \pm}\right|^{n}}\right)
$$

for any $(b, a) \in U_{\gamma}\left(h_{ \pm}^{n}\right)$ and by (7) we have

$$
\begin{aligned}
\delta_{(0, g)} * v(C(1 / R, R)) & \geq \mu^{* n}\left(U_{\gamma}\left(h_{+}^{n}\right)\right) v\left(C\left(|g|^{-1} 2\left|a_{ \pm}\right|^{-n} / R,|g|^{-1}\left|a_{ \pm}\right|^{-n} R / 2\right)\right) \\
& =C_{K_{ \pm}^{n}}^{-1} \delta_{(0, g)} * v\left(K_{ \pm}^{n}\right) .
\end{aligned}
$$

Take a radial function $\Phi_{0} \in C_{c}^{1}(V \backslash\{0\})$ such that $\Phi_{0} \geq \mathbf{1}_{C(1 / R, R)}$. Then, since $\left\{K_{ \pm}^{n}\right\}$ is a covering of $V \backslash\{0\}$, for any compact set $K$ in $V \backslash\{0\}$ there exists a finite number of compact sets of the type $K_{ \pm}^{n}$ covering $K$. Hence

$$
\delta_{(0, g)} * v(K) \leq C_{K} \delta_{(0, g)} * v\left(\Phi_{0}\right)
$$

for some constant $C_{K}$ and $g$ with small enough norm. Since $\delta_{(0, g)} * v\left(\Phi_{0}\right)>0$ when $|g|<1$ the above inequality holds for $|g|<1$ with possibly larger $C_{K}$. This proves that that $\frac{\delta_{(0, g)} * \nu}{\delta_{(0, g)} * \nu\left(\Phi_{0}\right)}$ is vaguely bounded as $|g|<1$, hence relatively vaguely compact.

Finally, one can easily see that in the non-lattice case the function $\Phi_{0}$ is exactly the one we are looking for. Indeed, we just set $\Phi=\Phi_{0}$ and to check the integral condition, since $\Phi$ is radial, we write

$$
\int_{G_{\mu}} \Phi(g u) \mathrm{d} g=\int_{G_{\mu}} \Phi\left(g g_{0} u\right) \mathrm{d} g=\int_{G_{\mu}} \Phi(g v) \mathrm{d} g
$$

for $g_{0} \in G_{\mu}$ with $\left|g_{0}\right|=|v| /|u|$. 
In the lattice case we have to modify slightly the function $\Phi_{0}$. Namely, take

$$
\Phi(u):=\int_{\substack{g^{\prime} \in G \\ 1<\left|g^{\prime}\right| \leq c}} \Phi_{0}\left(g^{\prime} u\right) m_{G}\left(\mathrm{~d} g^{\prime}\right) .
$$

Since $\Phi$ is, up to some constant, greater than $\mathbf{1}_{C(1 / R, R)}$ the first part of the Lemma is valid also for $\Phi$. Finally observe that

$$
\int_{G_{\mu}} \Phi(g u) \mathrm{d} g=\int_{G_{\mu}} \int_{g^{\prime} \in G, 1<\left|g^{\prime}\right| \leq c} \Phi_{0}\left(g^{\prime} g u\right) m_{G}\left(\mathrm{~d} g^{\prime}\right) \mathrm{d} g=\int_{G} \Phi_{0}\left(g^{\prime} u\right) m_{G}\left(\mathrm{~d} g^{\prime}\right)
$$

and applying the same argument as in the previous case we prove the Lemma.

Lemma 2 Every accumulation point of the family $\frac{\delta_{(0, g)} * v}{f_{\Phi}(g)}$ as $|g|$ goes to 0 is $G_{\mu}$-invariant. In consequence, for any $h \in G_{\mu}$

$$
\lim _{|g| \rightarrow 0, g \in G_{\mu}} \frac{f_{\Phi}(g h)}{f_{\Phi}(g)}=1 .
$$

Remark 1 Notice that since $\Phi$ is radial $f_{\Phi}$ is radial as well. Therefore, if we define $L\left(|g|^{-1}\right)=f_{\Phi}(g)$, the Lemma says that $L$ is slowly varying in the non-lattice case (compare with the slowly varying function $L$ defined in Theorem 2.1 in [5]).

Proof First we prove that if $\eta$ is an accumulation point of $\frac{\delta_{(0, g)} * v}{f_{\Phi}(g)}$ along a sequence belonging to $Z_{\mu}$, i.e. if

$$
\lim _{\left|z_{n}\right| \rightarrow 0, z_{n} \in Z_{\mu}} \frac{\delta_{\left(0, z_{n}\right)} * v(\phi)}{f_{\Phi}\left(z_{n}\right)}=\eta(\phi) \quad \forall \phi \in C_{c}(V \backslash\{0\}),
$$

then $\eta$ is $G_{\mu}$-invariant.

Fix a function $\phi \in C_{c}^{1}(V \backslash\{0\})$ and observe that for all $(b, a) \in H$ there exist a compact set $K \subset V \backslash\{0\}$ and a constant $C$ such that

$$
|\phi(g(a u+b))-\phi(g a u)| \leq C|g b| \mathbf{1}_{K}(g(a u))
$$

for $|g|$ satisfying $|g b|<\frac{1}{2} \operatorname{dist}(0, \operatorname{supp} \phi)$.

We claim that the function

$$
h(g)=\delta_{(0, g)} * \eta(\phi)=\lim _{n \rightarrow \infty} \frac{\delta_{\left(0, g z_{n}\right)} * v(\phi)}{f_{\Phi}\left(z_{n}\right)}
$$

defined on $G_{\mu}$ is $\bar{\mu}$-superharmonic. First observe that by (11)

$$
\frac{\left|\delta_{\left(0, z_{n}\right)} * \delta_{(b, a)} * v(\phi)-\delta_{\left(0, z_{n}\right)} * \delta_{(0, a)} * v(\phi)\right|}{f_{\Phi}\left(z_{n}\right)} \leq C\left|z_{n} b\right| \cdot \frac{\delta_{\left(0, z_{n}\right)} * v(a K)}{f_{\Phi}\left(z_{n}\right)} \stackrel{n \rightarrow \infty}{\longrightarrow} 0
$$


and then, by Fatou's Lemma we can write

$$
\begin{aligned}
\int_{G_{\mu}} h(g a) \bar{\mu}(\mathrm{d} a) & =\int_{H} h(g a) \mu(\mathrm{d} b, \mathrm{~d} a)=\int_{H} \delta_{(0, g a)} * \eta(\phi) \mu(\mathrm{d} b, \mathrm{~d} a) \\
& =\int_{H} \lim _{n \rightarrow \infty} \frac{\delta_{(0, g)} * \delta_{(0, a)} * \delta_{\left(0, z_{n}\right)} * v(\phi)}{f_{\Phi}\left(z_{n}\right)} \mu(\mathrm{d} b, \mathrm{~d} a) \\
& =\int_{H} \lim _{n \rightarrow \infty} \frac{\delta_{(0, g)} * \delta_{\left(0, z_{n}\right)} * \delta_{(b, a)} * v(\phi)}{f_{\Phi}\left(z_{n}\right)} \mu(\mathrm{d} b, \mathrm{~d} a) \\
& \leq \underset{n \rightarrow \infty}{\lim _{n \rightarrow \infty}} \frac{\delta_{(0, g)} * \delta_{\left(0, z_{n}\right)} * \mu * v(\phi)}{f_{\Phi}\left(z_{n}\right)}=\underline{\lim _{n \rightarrow \infty}} \frac{\delta_{(0, g)} * \delta_{\left(0, z_{n}\right)} * v(\phi)}{f_{\Phi}\left(z_{n}\right)} \\
& =\delta_{(0, g)} * \eta(\phi)=h(g) .
\end{aligned}
$$

Since $h$ is superharmonic and the right random walk on $G_{\mu}$ induced by $\bar{\mu}$ is recurrent the function $h$ is $\bar{\mu}$-harmonic. The Choquet-Deny theorem for $G$ (see [12], Theorem 3 , p. 52 and [17]) implies that $h$ is constant i.e. $\delta_{(0, g)} * \eta(\phi)=\eta(\phi)$ for any $g \in G_{\mu}$. This statement remains valid for any compactly supported continuous function $\phi$ on $V \backslash\{0\}$ not necessary differentiable.

To prove the Lemma in full generality we will use the structure of the group $G_{\mu}$ discussed in the Introduction. Take any accumulation point $\eta$ of $\delta_{(0, g)} * v / f_{\Phi}(g)$ as $|g| \rightarrow 0$ i.e. fix a sequence $\left\{g_{n}\right\}$ in $G_{\mu}$ such that

$$
\left|g_{n}\right| \rightarrow 0 \quad \text { and } \quad \frac{\delta_{\left(0, g_{n}\right)} * v}{f_{\Phi}\left(g_{n}\right)} \rightarrow \eta
$$

Since any element $g_{n} \in G_{\mu}$ can be written as $g_{n}=k_{n} z_{n}$ with $k_{n} \in K_{\mu}$ and $z_{n} \in Z_{\mu}$, extracting a sequence, we can suppose that

$$
k_{n} \rightarrow k \quad \text { and } \quad \delta_{\left(0, z_{n}\right)} * v / f_{\Phi}\left(z_{n}\right) \rightarrow \eta^{\prime}
$$

\section{Moreover}

$$
\begin{aligned}
& \left|\frac{\delta_{\left(0, k_{n}\right)} * \delta_{\left(0, z_{n}\right)} * v(\Phi)-\delta_{(0, k)} * \delta_{\left(0, z_{n}\right)} * v(\Phi)}{f_{\Phi}\left(z_{n}\right)}\right| \\
& \quad \leq \sup _{u}\left|\Phi\left(k_{n} u\right)-\Phi(k u)\right| \cdot \frac{\delta_{\left(0, z_{n}\right)} * v(A)}{f_{\Phi}\left(z_{n}\right)} \\
& \quad \leq C \sup _{u}\left|\Phi\left(k_{n} u\right)-\Phi(k u)\right| \stackrel{n \rightarrow \infty}{\longrightarrow} 0,
\end{aligned}
$$


where $A$ is an annulus containing all sets of the form $k^{-1} \operatorname{supp} \Phi, k_{n}^{-1} \operatorname{supp} \Phi$. Therefore we have

$$
\begin{aligned}
\lim _{n \rightarrow \infty} \frac{f_{\Phi}\left(g_{n}\right)}{f_{\Phi}\left(z_{n}\right)} & =\lim _{n \rightarrow \infty} \frac{\delta_{\left(0, k_{n}\right)} * \delta_{\left(0, z_{n}\right)} * v(\Phi)}{f_{\Phi}\left(z_{n}\right)} \\
& =\delta_{(0, k)} * \eta^{\prime}(\Phi)=\eta^{\prime}(\Phi)=\lim _{n \rightarrow \infty} \frac{\delta_{\left(0, z_{n}\right)} * v(\Phi)}{f_{\Phi}\left(z_{n}\right)}=1,
\end{aligned}
$$

and finally

$$
\eta=\lim _{n \rightarrow \infty} \frac{\delta_{\left(0, g_{n}\right)} * v}{f_{\Phi}\left(g_{n}\right)}=\lim _{n \rightarrow \infty} \frac{f_{\Phi}\left(z_{n}\right)}{f_{\Phi}\left(g_{n}\right)} \cdot \frac{\delta_{\left(0, k_{n}\right)} * \delta_{\left(0, z_{n}\right)} * v}{f_{\Phi}\left(z_{n}\right)}=\delta_{(0, k)} * \eta^{\prime}=\eta^{\prime}
$$

what proves the first part of the Lemma. For the second part we use exactly the same argument as above.

The first consequence of above lemma is the following

Corollary 3 Under assumptions of Theorem 1, the stationary measure v satisfies

$$
\int_{V} \frac{1}{(1+|u|)^{\gamma}} v(\mathrm{~d} u)<\infty
$$

In particular

$$
v(|u| \leq r) \leq C\left(1+r^{\gamma}\right)
$$

for any positive $\gamma$.

To proceed with the proof of our main result we need some an additional assumption on the measure $v$. Namely, without any loss of generality we may assume that

$$
\int \frac{1}{|u|^{\gamma}} v(\mathrm{~d} u)<\infty, \quad \text { for } \gamma \in(0,1)
$$

Indeed, there exists $b \in V$ such that $v_{b}=\delta_{(b, 0)} * v$ satisfies the condition above (see Lemma 4.4 in [5]). The measure $v_{b}$ is an invariant measure of $\delta_{(b, 0)} * \mu * \delta_{(-b, 0)}$ that also satisfies the assumptions of Theorem 1 and both measures $v_{b}$ and $v$ have the same behaviour at infinity, i.e.

$$
\lim _{|g| \rightarrow 0} \int(\phi(g(u+b))-\phi(g u)) v(\mathrm{~d} u)=0 .
$$

Therefore one can consider the measure $v_{b}$ instead of $v$, however to simplify our notation we will just write $v$ and assume that (14) is fulfilled. 


\subsection{Poisson equation}

For any $\phi \in C_{c}^{1}(V \backslash\{0\})$ we define $f_{\phi}(g):=\delta_{(0, g)} * v(\phi)$. To prove that the limit of $f_{\phi}$ exists as $|g|$ goes to 0 we consider $f_{\phi}$ as a solution of the following Poisson equation on $G_{\mu}$.

$$
\mathbb{E}\left[f_{\phi}(g M)\right]-f_{\phi}(g)=\psi_{\phi}(g),
$$

where the function $\psi_{\phi}$ is defined by the equation above.

The main tool to study this equation is the renewal theorem on $G$. Therefore, analogically to the situation on real line, we introduce the class of directly Riemann integrable on $G_{\mu}$ (compare [8]).

We say that a continuous function $\psi$ is directly Riemann integrable (dRi) if

$$
\sum_{n \in \mathbb{Z}} \sup _{\left\{g \in G_{\mu}, e^{n}<|g| \leq e^{n+1}\right\}}|\psi(g)|<\infty .
$$

Following the same way as in [5] (Lemma 4.8 and proof of Proposition 4.1) we can show that

Lemma 3 Assume that all the hypotheses of Theorem 1 and (14) are satisfied. Then for any Lipschitz function $\phi$ there exists $\zeta>0$ and constant $C$ such that

$$
\left|\psi_{\phi}(g)\right| \leq C \min \left\{|g|^{-\zeta},|g|^{\zeta}\right\},\left|f_{\phi}(g)\right| \leq C|g|^{-\zeta} .
$$

In particular the function $\psi_{\phi}$ is directly Riemann integrable.

Moreover, if $\phi=\Phi$ then

$$
\int_{G_{\mu}} \psi_{\Phi}(g) \mathrm{d} g=0
$$

Proof Since $\phi$ has a compact support, it is bounded by a some multiple of the function $|u|^{-\gamma}$. Therefore

$$
\left|f_{\phi}(g)\right| \leq \int_{V}|\phi(g u)| v(\mathrm{~d} u) \leq C_{0} \int \frac{1}{|g u|^{\gamma}} v(\mathrm{~d} u) \leq C_{1}|g|^{-\gamma}
$$

Now we will show that

$$
I(g):=\int_{H} \int_{V}|\phi(g a u)-\phi(g(a u+b))| v(\mathrm{~d} u) \mu(\mathrm{d} b, \mathrm{~d} a) \leq C\left(|g|^{-\zeta} \wedge|g|^{\zeta}\right)
$$

To get the first bound observe that by (17), (A4) and $\mu$ invariance of $\nu$, for $\gamma<\delta$ we have

$$
|I(g)| \leq \mathbb{E}\left[\left|f_{\phi}(g M)\right|\right]+\left|f_{\phi}(g)\right| \leq C_{0}\left(\mathbb{E}\left[|g M|^{-\gamma}\right]+|g|^{-\gamma}\right) \leq C_{1}|g|^{-\gamma} .
$$


In order to show the boundedness by a positive power of $|g|$, for $|g| \leq 1$ take $c_{0}=$ $1 / 2 \operatorname{dist}(\operatorname{supp} \phi, 0)$ and observe that

$$
\begin{aligned}
|I(g)|= & \int_{|g b| \leq c_{0}} \int|\phi(g(a u+b))-\phi(g a u)| v(\mathrm{~d} u) \mu(\mathrm{d} b, \mathrm{~d} a) \\
& +\int_{|g b|>c_{0}} \int|\phi(g(a u+b))-\phi(g a u)| v(\mathrm{~d} u) \mu(\mathrm{d} b, \mathrm{~d} a)=: I_{1}+I_{2} .
\end{aligned}
$$

To bound the first integral we will use the following inequality

$$
|\phi(g(a u+b))-\phi(g a u)| \leq C_{0}|g b| \mathbf{1}_{K}(g a u) \leq C_{1}|g b||g a u|^{-\gamma}
$$

valid for $|g b| \leq c_{0}$ (compare (11)). Then for $\gamma<\delta / 2$ we have

$$
\begin{aligned}
I_{1} & \leq C_{0} \int_{|g b| \leq c_{0}} \int|g b||g a u|^{-\gamma} \nu(\mathrm{d} u) \mu(\mathrm{d} b, \mathrm{~d} a) \\
& \leq C_{0} \int_{|g b| \leq c_{0}} c_{0}^{1-\delta / 2}|g|^{\delta / 2-\gamma}|b|^{\delta / 2}|a|^{-\gamma} \int|u|^{-\gamma} \nu(\mathrm{d} u) \mu(\mathrm{d} b, \mathrm{~d} a) \\
& \leq C_{1}|g|^{\delta / 2-\gamma} \int|b|^{\delta / 2}|a|^{-\gamma} \mu(\mathrm{d} b, \mathrm{~d} a) \leq C_{2}|g|^{\delta / 2-\gamma}
\end{aligned}
$$

where the last inequality follows from Schwartz inequality and assumptions of Theorem 1 . In order to estimate $I_{2}$ we can write

$$
I_{2} \leq \int_{|g b|>c_{0}} \int \phi(g a u) v(\mathrm{~d} u) \mu(\mathrm{d} b, \mathrm{~d} a)+\int_{|g b|>c_{0}} \int \phi(g(a u+b)) v(\mathrm{~d} u) \mu(\mathrm{d} b, \mathrm{~d} a)
$$

The first integral can be bounded by a multiple of

$$
\begin{aligned}
& \int_{|g b|>c_{0}} \int|g a u|^{-\gamma} \nu(\mathrm{d} u) \mu(\mathrm{d} b, \mathrm{~d} a) \\
& \quad \leq C_{0} \int_{|g b|>c_{0}}|g a|^{-\gamma} \mu(\mathrm{d} b, \mathrm{~d} a) \leq C_{1} \int_{|g b|>c_{0}}|g|^{\delta / 2-\gamma}|a|^{-\gamma}|b|^{\delta / 2} \mu(\mathrm{d} b, \mathrm{~d} a) \\
& \leq C_{1} \int|a|^{-\gamma}|b|^{\delta / 2} \mu(\mathrm{d} b, \mathrm{~d} a) \leq C_{2}|g|^{\delta / 2-\gamma}
\end{aligned}
$$

To estimate the second integral take $\gamma<\delta / 4$ and observe that if the support of $\phi$ is contained in the ball of radius $R$, then $\phi(g(a u+b)) \leq\|\phi\|_{\infty} \mathbf{1}_{|u| \leq(R+|b|) /|g a|}$, for 
$|g| \leq 1$, hence the integral can be estimated by

$$
\begin{aligned}
\int_{|g b|>c_{0}} & \int \mathbf{1}_{|u| \leq(R+|b|) /|g a|} v(\mathrm{~d} u) \mu(\mathrm{d} b, \mathrm{~d} a) \leq \int_{|g b|>c_{0}} \int\left(\frac{R+|b|}{|g a u|}\right)^{\gamma} v(\mathrm{~d} u) \mu(\mathrm{d} b, \mathrm{~d} a) \\
& \leq C_{0}|g|^{-\gamma} \int_{|g b|>c_{0}}(R+|b|)^{\gamma}|a|^{-\gamma} \mu(\mathrm{d} b, \mathrm{~d} a) \\
& \leq C_{1}|g|^{-\gamma} \int_{|g b|>c_{0}}\left(R^{\gamma}+|b|^{\gamma}\right)|a|^{-\gamma} \mu(\mathrm{d} b, \mathrm{~d} a) \\
& \leq C_{2}|g|^{-\gamma}\left(\int|g b|^{\delta / 4}|a|^{-\gamma} \mu(\mathrm{d} b, \mathrm{~d} a)+\int|g b|^{\delta / 4}|b|^{\gamma}|a|^{-\gamma} \mu(\mathrm{d} b, \mathrm{~d} a)\right) \\
& \leq C_{3}|g|^{\delta / 4-\gamma} .
\end{aligned}
$$

In summary, for $\zeta<\delta / 4$ we have $I(g) \leq C\left(|g|^{\zeta} \wedge|g|^{\zeta}\right)$ and since $\left|\psi_{\phi}(g)\right| \leq I(g)$ we get also the desired inequality for $\psi_{\phi}$. Moreover, since $I$ is $m_{G_{\mu}}$-integrable by the Fubini theorem we can interchange the order of integration in the definition of $\psi_{\Phi}$ and by Lemma 1 get $\int_{G_{\mu}} \psi_{\Phi}(g) \mathrm{d} g=0$.

At this moment we only know that $\int_{G_{\mu}} \psi_{\phi}(g) \mathrm{d} g=0$ for $\phi=\Phi$ but later on, using the renewal theorem on $G$, we will prove that this holds for general $\phi \in C_{c}^{1}(V \backslash\{0\})$.

Proposition 1 Let $\psi$ be a function on $G_{\mu}$ satisfying $|\psi(g)| \leq \min \left\{|g|^{-\zeta},|g|^{\zeta}\right\}$ such that $\int_{G_{\mu}} \psi(g) \mathrm{d} g=0$. Then the function

$$
\bar{\psi}(g):=\int_{G_{\mu}} \psi(h) \mathbf{1}_{\{|h|>|g|\}} \mathrm{d} h
$$

satisfies

$$
|\bar{\psi}(g)| \leq C\left(|g|^{-\zeta} \wedge|g|^{\zeta}\right)
$$

hence it is directly Riemann integrable. Furthermore

$$
\int_{G_{\mu}} \bar{\psi}(g) \mathrm{d} g=\int_{G_{\mu}} \log |g| \psi(g) \mathrm{d} g
$$

Proof For $|g| \geq 1$, by (16), we get

$$
|\bar{\psi}(g)| \leq \int_{|h|>|g|}|\psi(h)| \mathrm{d} h \leq \int_{|h|>|g|}|h|^{-\zeta} \mathrm{d} h=|g|^{-\zeta} \int_{|h|>1}|h|^{-\zeta} \mathrm{d} h \leq C|g|^{-\zeta}
$$


and similarly for $|g|<1$ we have

$$
|\bar{\psi}(g)|=\left|\int_{|h|>|g|} \psi(h) \mathrm{d} h\right|=\left|\int_{|h| \leq|g|} \psi(h) \mathrm{d} h\right| \leq|g|^{\zeta} \int_{|h| \leq 1}|h|^{\zeta} \mathrm{d} h \leq C|g|^{\zeta} .
$$

Moreover, we can easily compute the integral of $\bar{\psi}$

$$
\begin{aligned}
\int_{G_{\mu}} \bar{\psi}(g) \mathrm{d} g & =\int_{|g| \geq 1} \int_{|h|>|g|} \psi(h) \mathrm{d} h \mathrm{~d} g-\int_{|g|<1} \int_{|h| \leq|g|} \psi(h) \mathrm{d} h \mathrm{~d} g \\
& =\int_{|h|>1} \int_{1 \leq|g|<|h|} \mathrm{d} g \psi(g) \mathrm{d} h-\int_{|h| \leq 1} \int_{|h| \leq|g|<1} \mathrm{~d} g \psi(h) \mathrm{d} h \\
& =\int_{|h|>1} \log |h| \psi(h) \mathrm{d} h+\int_{|h| \leq 1} \log |h| \psi(h) \mathrm{d} h=\int_{G_{\mu}} \log |g| \psi(g) \mathrm{d} g .
\end{aligned}
$$

\subsection{Proof of Theorem 1}

Now we are going to apply a technique due to Durrett and Liggett [9]. They described asymptotic behaviour of positive solutions of a Poisson equation not solving explicitly the equation as in $[5,6]$, but applying the duality lemma and reducing the problem to the classical renewal equation.

On the group $G_{\mu}$ we define two random walks, the right random walk $R_{i}=$ $M_{1} \cdots M_{i}$ and the left one $L_{i}=M_{i} \cdots M_{1}$. We define a family of weakly increasing ladder times

$$
\begin{aligned}
T_{0} & =0 \\
T_{i} & =\inf \left\{n>T_{i-1}:\left|R_{n}\right| \geq\left|R_{T_{i-1}}\right|\right\} \\
& =\inf \left\{n>T_{i-1}:\left|L_{n}\right| \geq\left|L_{T_{i-1}}\right|\right\} .
\end{aligned}
$$

Now we can define a new process $\left\{R_{T_{n}}\right\}$ which is also a right random walk on $G_{\mu}$ with increments distributed according to $R_{T_{1}}$. One can observe that the closed subgroup $G^{\prime}$ generated by the $\left\{R_{T_{n}}\right\}$ is exactly $G_{\mu}$. Indeed, any $g_{+} \in \operatorname{supp} \bar{\mu}$ such that $\left|g_{+}\right| \geq 1$ belongs to $G^{\prime}$. Moreover, if $g_{-} \in \operatorname{supp} \mu$ with $\left|g_{-}\right|<1$ then we can find a natural number $k$ satisfying $\left|g_{-} g_{+}^{k-1}\right|<1$ and $\left|g_{-} g_{+}^{k}\right| \geq 1$. Therefore, $g_{-} g_{+}^{k}$ is in $G^{\prime}$ hence also $g_{-}$.

We define a new stopping time $\tau$ by

$$
\tau=\inf \left\{k:\left|R_{k}\right|<1\right\}
$$

and construct a new Poisson equation with a drift: 
Lemma 4 Suppose that the functions $f$ and $\psi$ defined on $G_{\mu}$ satisfy the Poisson equation

$$
\mathbb{E}[f(g M)]-f(g)=\psi(g)
$$

and $|f(g)| \leq|g|^{-\zeta},|\psi(g)| \leq|g|^{-\zeta} \wedge|g|^{\zeta}$, for some $0<\zeta<\delta$. Then $f$ satisfies a new Poisson equation

$$
\mathbb{E}\left[f\left(g R_{\tau}\right)\right]-f(g)=\sum_{n=0}^{\infty} \mathbb{E}\left[\psi\left(g R_{T_{n}}\right)\right]
$$

Moreover, for $h \in G_{\mu}$

$$
\mathbb{E}\left[\int_{\left|R_{\tau}\right| \leq|g|<1} f(g h) \mathrm{d} g\right]=\sum_{n=0}^{\infty} \mathbb{E}\left[\bar{\psi}\left(h R_{T_{n}}\right)\right] .
$$

Proof First notice that

$$
\mathscr{M}_{n}(g):=f\left(g M_{1} \cdots M_{n}\right)-\sum_{i=0}^{n-1} \psi\left(g M_{1} \cdots M_{i}\right)
$$

forms a martingale with respect to the filtration induced by the sequence $\left\{M_{n}\right\}_{n \in \mathbb{N}}$. From the optional stopping theorem we obtain

$$
\mathbb{E}\left[\mathscr{M}_{n \wedge \tau}(g)\right]=f(g)
$$

By the assumption (A4) and (3.6a) of Chap. XII in [10]

$$
\mathbb{E}\left[\left|R_{\tau}\right|^{-\delta}\right]=\mathbb{E}\left[e^{-\delta S_{\tau}}\right]<\infty
$$

where $S_{i}=\log \left|R_{i}\right|=\sum_{0 \leq k \leq i} \log \left|M_{k}\right|$. Therefore by Lemma 3

$$
\mathbb{E}\left[\left|f\left(g R_{\tau}\right)\right|\right] \leq \mathbb{E}\left[|g|^{-\zeta} e^{-\zeta S_{\tau}}\right]<\infty
$$

Moreover the duality lemma and the renewal theorem on $\mathbb{R}$ imply

$$
\mathbb{E}\left[\sum_{i=0}^{\tau-1}\left|\psi\left(g R_{i}\right)\right|\right] \leq \mathbb{E}\left[\sum_{i=0}^{\tau-1} e^{-\zeta|\log | g\left|+S_{i}\right|}\right]=\mathbb{E}\left[\sum_{i=0}^{\infty} e^{-\zeta|\log | g\left|+S_{T_{i}}\right|}\right]<\infty
$$


Now by the Lebesgue theorem we can pass to the limit as $n$ goes to infinity in (22) and get

$$
\mathbb{E}\left[f\left(g R_{\tau}\right)\right]-\mathbb{E}\left[\sum_{i=0}^{\tau-1} \psi\left(g R_{i}\right)\right]=\mathbb{E}\left[M_{\tau}(g)\right]=f(g) .
$$

Observe that from duality lemma

$$
\mathbb{E}\left[\sum_{i=0}^{\tau-1} \psi\left(g R_{i}\right)\right]=\sum_{n=0}^{\infty} \mathbb{E}\left[\psi\left(g L_{T_{n}}\right)\right]=\sum_{n=0}^{\infty} \mathbb{E}\left[\psi\left(g R_{T_{n}}\right)\right]
$$

Comparing (24) with (25) we obtain

$$
\mathbb{E}\left[f\left(g R_{\tau}\right)\right]-f(g)=\sum_{n=0}^{\infty} \mathbb{E}\left[\psi\left(g R_{T_{n}}\right)\right]
$$

Fix now an element $h \in G_{\mu}$. Integrating both sides of above equation we get

$$
\int_{|g| \geq|h|} \mathbb{E}\left[f\left(g R_{\tau}\right)\right] \mathrm{d} g-\int_{|g| \geq|h|} f(g) \mathrm{d} g=\int_{|g| \geq|h|} \sum_{n=0}^{\infty} \mathbb{E}\left[\psi\left(g R_{T_{n}}\right)\right] \mathrm{d} g,
$$

what can be rewritten as

$$
\mathbb{E}\left[\int_{|h|>|g| \geq\left|h R_{\tau}\right|} f(g) \mathrm{d} g\right]=\int_{|g| \geq|h|} \sum_{n=0}^{\infty} \mathbb{E}\left[\psi\left(g R_{T_{n}}\right)\right] \mathrm{d} g
$$

by Fubini theorem and Lemma 3

$$
\begin{aligned}
\mathbb{E}\left[\int_{1>|g| \geq\left|R_{\tau}\right|} f(g h) \mathrm{d} g\right] & =\sum_{n=0}^{\infty} \int_{|g| \geq|h|} \mathbb{E}\left[\psi\left(g R_{T_{n}}\right)\right] \mathrm{d} g \\
& =\sum_{n=0}^{\infty} \mathbb{E}\left[\int_{|g| \geq|h|} \psi\left(g R_{T_{n}}\right) \mathrm{d} g\right] \\
& =\sum_{n=0}^{\infty} \mathbb{E}\left[\bar{\psi}\left(h R_{T_{n}}\right)\right]
\end{aligned}
$$

Now we are ready to prove our main result. 
Proof (Proof of the Main Theorem 1) First we shall show that the theorem holds for the special function $\phi=\Phi$ defined in the Lemma 1 . In other words we need to prove that $f_{\Phi}(h)$ has a limit as $|h| \rightarrow 0$. By (16) and Lemma 4 we obtain

$$
\mathbb{E}\left[\int_{R_{\tau} \leq|g|<1} f_{\Phi}(g h) \mathrm{d} g\right]=\sum_{n=0}^{\infty} \mathbb{E}\left[\overline{\psi_{\Phi}}\left(h R_{T_{n}}\right)\right]
$$

Since $f_{\Phi}$ is radial, bounded on compact sets, and, by Lemma $2, f_{\Phi}(g h) / f_{\Phi}(h) \rightarrow 1$, for any $\gamma>0$ we can find $C$ such that $f_{\Phi}(g h) / f_{\Phi}(h)<C|g|^{-\gamma}$ for $|g|,|h| \leq 1$ (cf. Potter's theorem [2, Theorem 1.5.6]). By (23), Lebesgue's Dominated Convergence Theorem and the renewal theorem for group $G$ (see Theorem A.1 in Appendix in [8] for more details) apply to

$$
f_{\Phi}(h) \cdot \mathbb{E}\left[\int_{\left|R_{\tau}\right| \leq|g|<1} \frac{f_{\Phi}(g h)}{f_{\Phi}(h)} \mathrm{d} g\right]=\sum_{n=0}^{\infty} \mathbb{E}\left[\overline{\psi_{\Phi}}\left(h R_{T_{n}}\right)\right]
$$

we obtain

$$
\lim _{|h| \rightarrow 0, h \in G_{\mu}} f_{\Phi}(h) \mathbb{E}\left[-S_{\tau}\right]=\frac{\int_{G_{\mu}} \overline{\psi_{\Phi}}(g) \mathrm{d} g}{\mathbb{E}\left[S_{T_{1}}\right]}
$$

Hence for $\sigma^{2}:=\operatorname{Var}(\log |M|)=2 \mathbb{E}\left[-S_{\tau}\right] \mathbb{E}\left[S_{T_{1}}\right]$ (see the proof of T18.1 on page 196 in [18])

$$
\lim _{|h| \rightarrow 0, h \in G_{\mu}} f_{\Phi}(h)=\frac{2}{\sigma^{2}} \int_{G_{\mu}} \overline{\psi_{\Phi}}(g) \mathrm{d} g=\frac{2}{\sigma^{2}} \int_{G_{\mu}} \log |g| \psi_{\Phi}(g) \mathrm{d} g .
$$

Moreover the same argument as in Theorem 1.5 [5] proves that the limit above is strictly positive.

Now take arbitrary $\phi \in C_{c}^{1}(V \backslash\{0\})$. In view of Lemma 1 the family $\left\{\delta_{(0, g)} * v\right\}$ is vaguely compact and any accumulation point, as $|g| \rightarrow 0$, is $G_{\mu}$-invariant. This implies that the set of real numbers $f_{\phi}(g)$ is bounded and any accumulation point $L$ of $f_{\phi}(h)$ as $h \in Z_{\mu}$ is also accumulation point of shifted sequence i.e. if $f_{\phi}\left(h_{n}\right) \rightarrow L$ then also $f_{\phi}\left(g h_{n}\right) \rightarrow L$ for any $g \in G_{\mu}$. However, we would like to say that also $f_{\phi}\left(h_{n} g\right) \rightarrow L$. Take any limit point $L^{\prime}$ of a sequence $f_{\phi}\left(h_{n} g\right)$. Then, since the commutator $\left[h_{n}, g\right]=h_{n} g h_{n}^{-1} g^{-1}$ belongs to the compact group $O_{\mu}$, we can extract a subsequence such that

$$
\left[g, h_{n_{m}}\right] \rightarrow k \quad \text { and } \quad f_{\phi}\left(h_{n_{m}} g\right) \rightarrow L^{\prime}
$$


By the same argument as (12) in the proof of Lemma 2 we get

$$
\lim _{m \rightarrow \infty}\left|f_{\phi}\left(k g h_{n_{m}}\right)-f_{\phi}\left(\left[h_{n_{m}} g\right] g h_{n_{m}}\right)\right|=0 .
$$

Thus,

$$
L=\lim _{m \rightarrow \infty} f_{\phi}\left(k g h_{n_{m}}\right)=\lim _{m \rightarrow \infty} f_{\phi}\left(\left[h_{n_{m}} g\right] g h_{n_{m}}\right)=\lim _{m \rightarrow \infty} f_{\phi}\left(h_{n_{m}} g\right)=L^{\prime} .
$$

We are now ready to show that $\int_{G_{\mu}} \psi_{\phi}(g) \mathrm{d} g=0$. From (20) we get

$$
\mathbb{E}\left[f_{\phi}\left(h_{n} R_{\tau}\right)\right]-f_{\phi}\left(h_{n}\right)=\sum_{k=0}^{\infty} \mathbb{E}\left[\psi_{\phi}\left(h_{n} R_{T_{k}}\right)\right] .
$$

By Lemma 3 the function $\psi_{\phi}$ defined on the group $G_{\mu}$ is directly Riemann integrable therefore we can apply the renewal theorem for $G_{\mu}$ to the right hand side of the equation above (see Appendix in [8] for more details) and the Lebesgue theorem to the left hand side and obtain

$$
0=\mathbb{E}[L]-L=\frac{\int_{G_{\mu}} \psi_{\phi}(g) \mathrm{d} g}{\mathbb{E}\left[S_{T_{1}}\right]},
$$

since the closed subgroup generated by the law of $R_{T_{1}}$ is $G_{\mu}$.

By the equation (21) we have

$$
\lim _{k \rightarrow \infty} \mathbb{E}\left[\int_{R_{\tau} \leq|g|<1} f\left(g h_{k}\right) \mathrm{d} g\right]=\lim _{k \rightarrow \infty} \sum_{n=0}^{\infty} \mathbb{E}\left[\bar{\psi}\left(h_{k} R_{T_{n}}\right)\right] .
$$

By Proposition $1, \overline{\psi_{\phi}}$ is directly Riemann integrable. Using the Lebesgue theorem to the left hand side and renewal theorem to right one we get

$$
L \cdot \mathbb{E}\left[-S_{\tau}\right]=\frac{\int_{G_{\mu}} \overline{\psi_{\phi}}(g) \mathrm{d} g}{\mathbb{E}\left[S_{T_{1}}\right]} .
$$

The above equation shows us that $L$ does not depends on the particular choice of the sequence $h_{n}$, hence

$$
\lim _{|g| \rightarrow 0, g \in G_{\mu}} \delta_{(0, g)} * v(\phi)=\frac{2}{\sigma^{2}} \int_{G_{\mu}} \log |g| \psi_{\phi}(g) \mathrm{d} g .
$$

By Lemma 1 the set $\delta_{(0, g)} * v$ is relatively weakly compact as $|g| \rightarrow 0$. Take any accumulation point $\Lambda$, then it is uniquely determined on the class $C_{c}^{1}(V \backslash\{0\})$ therefore, by density, also on $C_{c}(V \backslash\{0\})$. It yields that $\Lambda$ is a limit of $\delta_{(0, g)} * v$ as $|g| \rightarrow 0$. 
Moreover, since for every $\delta_{(0, g)} * v$ is nonnegative measure also $\Lambda$ is nonnegative. To see where the product formula comes from observe that for any Borel set $B_{2} \subset \Sigma_{1}$ the mapping

$$
B_{1} \rightarrow \Lambda\left(B_{1} \times B_{2}\right)
$$

defined on Borel sets on $A_{\mu}$ is a $A_{\mu}$-invariant measure, hence there exists a constant $\sigma\left(B_{2}\right)$ depending on $B_{2}$ such that

$$
\Lambda\left(B_{1} \times B_{2}\right)=\sigma\left(B_{2}\right) \cdot m_{A_{\mu}}\left(B_{1}\right)
$$

On the other side, for any Borel set $B_{1}$ on $A_{\mu}$ with positive Haar measure

$$
B_{2} \rightarrow \frac{\Lambda\left(B_{1} \times B_{2}\right)}{m_{A_{\mu}}\left(B_{1}\right)}
$$

define a finite positive measure that is $O_{\mu}$ invariant. This finishes the proof, since the measure $\Lambda$ is determined by the value on the sets of the form $B_{1} \times B_{2}$.

Acknowledgments The results of this paper are part of the author's $\mathrm{PhD}$ thesis, written under the supervision of Dariusz Buraczewski at the University of Wroclaw. I wish to thank him for many stimulating conversation, suggestions and corrections incorporated in this paper. Part of this paper was written when I was staying at the Graz University of Technology at the invitation of Wolfgang Woess whom I would like to thank for help and hospitality. I would also like to thank anonymous referee for valuable comments that allowed to shortened the proof and write it more clearly.

Open Access This article is distributed under the terms of the Creative Commons Attribution License which permits any use, distribution, and reproduction in any medium, provided the original author(s) and the source are credited.

\section{References}

1. Babillot, M., Bougerol, P., Élie, L.: The random difference equation $X_{n}=A_{n} X_{n-1}+B_{n}$ in the critical case. Ann. Probab. 25, 478-493 (1997)

2. Bingham, N.H., Goldie, C.M., Teugels, J.T.: Regular Variation. Cambridge University Press, Cambridge (1989)

3. Brofferio, S.: How a centered random walk on the affine group goes to infinity. Ann. Inst. H. Poincar 39, 371-384 (2003)

4. Brofferio, S.: Speed of stochastic locally contracting systems. Ann. Probab. 31, 2040-2067 (2003)

5. Brofferio, S., Buraczewski, D., Damek, E.: On the invariant measure of the random difference equation $X_{n}=A_{n} X_{n-1}+B_{n}$ in the critical case. Ann. Inst. H. Poincaré Probab. Statist. 48, 377-395 (2012)

6. Buraczewski, D.: On invariant measures of stochastic recursions in a critical case. Ann. Appl. Probab. 17, 1245-1272 (2007)

7. Buraczewski, D., Damek, E., Guivarc'h, Y.: Convergence to stable laws for a class of multidimensional stochastic recursions. Probab. Theory Relat. Fields 148, 333-402 (2010)

8. Buraczewski, D., Damek, E., Guivarch, Y., Hulanicki, A., Urban, R.: Tail-homogeneity of stationary measures for some multidimensional stochastic recursions. Probab. Theory Relat. Fields 145, 385420 (2009)

9. Durrett, R., Liggett, T.M.: Fixed points of the smoothing transformation. Z. Wahrsch. Verw. Gebiete 64, 275-301 (1983)

10. Feller, W.: An introduction to probability theory and its application II., 2nd edn. Wiley, New York (1971) 
11. Goldie, C.M.: Implicit renewal theory and tails of solutions of random equations. Ann. Appl. Probab. 1, 126-166 (1991)

12. Guivarch, Y.: Extension dun théorème de Choquet-Deny à une classe de groupes non abéliens. Asterisque 4, 41-60 (1973)

13. Guivarch, Y.: Heavy tail properties of stationary solutions of multidimensional stochastic recursions. IMS Lecture Notes-Monograph Series. Dyn. Stoch. 48, 85-99 (2006)

14. Kesten, H.: Random difference equations and renewal theory for products of random matrices. Acta Math. 131, 207-248 (1973)

15. Le Page, É.: Théorèmes de renouvellement pour les produits de matrices aléatoires. Équations aux différences aléatoires. Séminaires de probabilités Rennes 1983, p.116, Univ. Rennes I, Rennes (1983)

16. Port, S.C., Stone, C.J.: Potential theory for random walks on abelian groups. Acta Math. 122, 19115 (1969)

17. Raugi, A.: A general Choquet-Deny theorem for nilpotent groups. Ann. I.H. Poincaré-PR 40, 677683 (2004)

18. Spitzer, F.: Principles of Random Walk, 2nd edn. Springer, New York (1976) 